\title{
Study of alterations in pharmacokinetics and pharmacodynamics of Saxagliptin in the presence of Rutin: An interaction study in rats
}

\author{
Naga Raju Kandukoori, Pavani Uppu, Narsimha Reddy Yellu* \\ University College of Pharmaceutical Sciences, Kakatiya University, Warangal, Telangana-506009, India.
}

\section{ARTICLE INFO \\ Received on: 20/07/2020 \\ Accepted on: 19/09/2020 \\ Available online: 05/11/2020}

\section{Key words:}

Rutin, Saxagliptin,

pharmacokinetics,

pharmacodynamics and

CYP 3 A4.

\begin{abstract}
The metabolism of most of the antidiabetic drugs is influenced by cytochrome P-450 (CYP) enzymes in the human body. The activity of these CYP enzymes is affected by the phytoconstituents which are present in vegetables, fruits and some ayurvedic preparations. In the present study, the effect of Rutin on the metabolism of Saxagliptin was studied. The drug was administered alone as well as in combination with Rutin to male albino Wistar rats for 8 days. The blood samples were collected periodically and all the pharmacokinetic parameters, like maximum concentration of drug in serum $\left(C_{\max }\right.$, time required to get $C_{\max }\left(t_{\max }\right)$, area under curve (AUC), mean residence time, volume of distribution $\left(V_{\mathrm{d}}\right)$, and total drug clearance $\left(\mathrm{Cl}_{\mathrm{T}}\right)$, were determined and compared. The glucose levels were also estimated and compared. From the results, it could be concluded that Rutin has induced the activity of CYP 3 A4 enzyme, thereby increasing the metabolism of the drug which ultimately lead to the decrease in $C_{\max }$ and AUC. Rutin could lower the antidiabetic effect of the drug.
\end{abstract}

\section{INTRODUCTION}

Cytochrome P-450 (CYP) enzymes present in the human body play a vital role in deciding the bioavailability of administered drugs. These enzymes will metabolize the drug substrates and change their forms. The altered forms may be active or inactive. Most of the antidiabetic drugs are substrates for these microsomal CYP enzymes. As the administered drugs are metabolized by these enzymes, their pharmacokinetics and pharmacodynamics will be affected. Many substances of natural or synthetic origin may inhibit or induce the activity of CYP enzymes, thereby affecting the extent of metabolism of the substrate drugs. If these substances are administered along with antidiabetic drugs, the final therapeutic efficacy of drugs will be altered (Wanwimolruk and Prachayasittikul, 2014). Some phytoconstituents available in dietary fruits, vegetables, and other plant sources show an effect on CYP enzymes by inhibiting or inducing them. These phytoconstituents also show therapeutic activities. If the

\section{"Corresponding Author}

Narsimha Reddy Yellu, University College of Pharmaceutical Sciences, Kakatiya University, Warangal, Telangana-506009.

E-mail:ynrku@kakatiya.ac.in phytochemicals with antidiabetic activity and effector activity toward CYP enzymes are ingested along with antidiabetic drugs, they alter the pharmacokinetics and pharmacodynamics of the antidiabetic drugs (Gaikwad et al., 2014).

Flavonoids are a group of polyphenols that are abundantly available in many dietary items and plants (Yu et al., 2011). Rutin (3, 3',4',5,7-pentahydroxyflavone 3-rhamnose glucoside) is a flavanol. It is found abundantly in plants, such as passion flower and buckwheat, and also in fruits like apples, oranges, grapes, lemons, berries, and peaches. It shows antidiabetic activity and it induces CYP 3A4 activity (Ganeshpurkar and Saluja, 2017; Aba and Asuzu, 2018). Saxagliptin is an orally active hypoglycemic of the new dipeptidyl peptidase-4 (DPP-4) inhibitor class of drugs. It is metabolized by CYP 3A4 and CYP 3A5 enzymes (Sowjanya et al., 2017; Gao et al., 2013).

Diabetic patients may unknowingly ingest fruits and vegetables which contain Rutin. Some diabetic people may also prefer other ayurvedic products containing Rutin as active ingredient and these ayurvedic products may be ingested along with the prescribed synthetic drug, like Saxagliptin. If Rutin is ingested along with Saxagliptin, the metabolism of the drug may be enhanced, leading to an alteration of the bioavailable form of drug, and also there is a chance of alteration in the therapeutic 
activity of drug. So, there is a need to study the consequences due to the interaction of Rutin with Saxagliptin. In this research study, the interaction between Rutin and Saxagliptin is studied using rat models and the alterations in pharmacokinetics and pharmacodynamics of drug are reported.

\section{MATERIALS AND METHODS}

\section{Drugs and chemicals}

Saxagliptin and repaglinide (gifted by Glenmark Pharmaceuticals Limited, Mumbai), Rutin (purchased from Kshipra Biotech Pvt. Ltd.), streptozotocin (purchased from Sisco Research Labs, Mumbai), methanol (HPLC-grade), citric acid, isoflurane, and sodium bicarbonate (purchased from Merck Labs) were used in the study.

\section{Animals used in the study}

Male Wistar rats (8-9 weeks old) weighing 230$260 \mathrm{~g}$ were used in the study. In most of the related literature studies, male rats are preferred because of their stable hormonal status. This in-vivo experimental protocol was approved by the Institutional Animal Ethical committee, Kakatiya University, Warangal (approval no. IAEC/05/UCPSc/KU/2020). Wistar rats were procured from a registered vendor. Animals were hosted in a controlled environment in polypropylene cages as per CPCSEA guidelines and fed a standard rat pellet diet and water ad libitum. The rats were fasted over night before starting the study (Jyothi et al., 2017; Neearti, 2012).

\section{Chromatographic analysis of Saxagliptin}

The serum concentration of Saxagliptin was quantified by a validated Ultra-Fast Liquid Chromatography coupled with photodiode array detection (Shimadzu Corporation, Japan). This modified reverse phase (RP) high-performance liquid chromatography (HPLC system consisted of binary LC-20AD pumps with a micro-gradient mixer. RP C18 column $(250 \times$ $4.6 \mathrm{~mm}, 5 \mu \mathrm{m}$, Phenomenex Luna) was used as the stationary phase. The mobile phase used in the study was a mixture of methanol and distilled water (adjusted to $\mathrm{pH} 3.15$ ) in the ratio of 50:50 at a flow rate of $1 \mathrm{ml} /$ minute. The mobile phase was degassed using an ultra sonicator and filtered through a 0.22 $\mu \mathrm{m}$ membrane filter. The detection of Saxagliptin was carried out at a wavelength of $220 \mathrm{~nm}$. Repaglinide was used as an internal standard. The total run time of the analysis was 10 minutes. Lab solutions software was used for carrying out all analysis operations and the same software was also used for the interpretation of the analyzed data.

\section{Sample preparations for HPLC analysis}

$100 \mu \mathrm{l}$ of test serum was added to $100 \mu \mathrm{l}$ of internal standard in a centrifuge tube. Repaglinide solution $(10 \mu \mathrm{g} / \mathrm{ml})$ was used as an internal standard. This mixture was shaken for 1 minute. To this mixture, $100 \mu \mathrm{l}$ of methanol was added for precipitation. The resultant was vortexed for 1 minute and centrifuged for 20 minutes at 3,000 rpm. Then, the supernatant was collected and filtered. $20 \mu \mathrm{l}$ of the filtrate was injected in to the HPLC system for analysis.
The precision and accuracy of the method were determined by analyzing the quality control samples at three concentrations: 2 , 10 , and $100 \mu \mathrm{g} / \mathrm{ml}$. An analysis was carried out in three replicates. Three sets of quality control samples were analyzed to get intraday precision data, whereas the samples were analyzed on three consecutive days to obtain inter-day precision data.

\section{Experimental design for in-vivo studies}

\section{Pharmacokinetic interaction study in normal rats}

The rats were divided in to three groups (each group had six rats; $n=6$ ) after overnight fasting. Group 1 was administered with Saxagliptin. The rats in Group 2 were administered with Rutin, followed by Saxagliptin in a single-dose interaction (SDI) study. Group 3 was given Rutin for 7 days consecutively and on the 8th day, they were administered with Rutin, followed by Saxagliptin, in a multi-dose injection (MDI) study. In all the groups, Saxagliptin was administered at a dose of $8 \mathrm{mg} / \mathrm{kg}$ body weight (Sowjanya et al., 2017) and Rutin was administered at a dose of $20 \mathrm{mg} / \mathrm{kg}$ body weight (Ganeshpurkar and Saluja, 2017). In each group, after the administration of Saxagliptin, the rats were anaesthetized by allowing them to inhale isoflurane for 2 minutes. Then, the blood samples were collected from the retro orbital plexus using heparinized capillary tubes at particular predetermined time points: $0,0.5,1,1.5$, $2,4,8,12$, and 24 hours. After collecting the blood samples, double the volume was replaced with normal saline. The collected blood samples were centrifuged in a cooling centrifuge (Heraeus Biofuge Fresco centrifuge, Germany). Then, the serum was separated from the centrifuged blood samples and the separated serum samples were stored at $-80^{\circ} \mathrm{C}$ until analysis (Jyothi et al., 2017; Neearti, 2012).

\section{Pharmacokinetic interaction study in diabetic rats}

\section{Induction of diabetes in rats}

Freshly prepared streptozotocin solution in $\mathrm{pH} 4.5$ citrate buffer was given to the rats (after overnight fasting) via the intraperitoneal (i.p.) route at a dose of $55 \mathrm{mg} / \mathrm{kg}$. Dextrose solution was given to the rats at different time points for avoiding sudden hypoglycemia. First, 20\% dextrose solution was given via the i.p route after 4-6 hours of streptozotocin injection and then the rats were fed orally with $50 \%$ dextrose solution up to 24 hours. Blood samples were collected from the retro orbital plexus after 72 hours of streptozotocin injection. The serum glucose levels were estimated by the peroxidase (POD) glucose oxidase (GOD) method. The rats having blood glucose levels more than $250 \mathrm{mg} / \mathrm{dl}$ were considered as diabetic and were used for study (Jyothi et al., 2017).

The diabetic rats (after overnight fasting) were grouped and treated same as in the study with normal rats. Here also, the blood samples were collected and the separated serum samples were stored at $-80^{\circ} \mathrm{C}$ until analysis.

After attaining the serum drug concentration values against time points, the pharmacokinetic parameters, like maximum concentration of drug in serum $\left(C_{\max }\right)$, time required to get $C_{\max }\left(t_{\max )}\right.$, area under curve (AUC), mean residence time (MRT), volume of distribution $\left(V_{\mathrm{d}}\right)$, and total drug clearance $\left(C l_{\mathrm{T}}\right)$, were calculated using the Kinetica TM software (version 4.4.1, Thermo Fisher Scientific Corporation, USA). 


\section{Pharmacodynamic interaction study in diabetic rats}

The diabetic rats (after overnight fasting) were divided into three groups (each consisting of six rats; $n=6$ ). Group 1 was administered with Saxagliptin only. Group 2 was given Rutin, followed by Saxagliptin (SDI study). Group 3 was administered with Rutin for 7 days and on the 8th day, the rats were given Rutin, followed by Saxagliptin (MDI study). Blood samples were collected from the retro orbital plexus at different time points. The blood glucose levels were estimated using the GOD-POD method.

The mean glucose levels and percentage reduction in blood glucose were estimated in each group and compared with each other.

$\%$ Glucose reduction at $t$ hour $=\left[\left(G_{0}-G_{\mathrm{t}}\right) / G_{0}\right] \times 100$

where $G_{\mathrm{t}}=$ mean glucose level at $t$ hour

$G_{0}=$ mean glucose level at 0 hour (Neearti, 2012)

Table 1. Pharmacokinetic parameters of Saxagliptin in normal rats.

\begin{tabular}{lccc}
\hline PK parameter & SXG & SXG + Rutin (SDI) & SXG + Rutin (MDI) \\
\hline$C_{\max }(\mu \mathrm{g} / \mathrm{ml})$ & $5.46 \pm 0.23$ & $4.96 \pm 0.32^{*}$ & $4.82 \pm 0.29^{* *}$ \\
$T_{\max }($ hour $)$ & $2.0 \pm 0$ & $2.0 \pm 0$ & $2.0 \pm 0$ \\
$\mathrm{AUC}_{0-\mathrm{n}}(\mu \mathrm{g}$. hour$/ \mathrm{ml})$ & $39.2 \pm 2.42$ & $31.4 \pm 1.12^{*}$ & $28.6 \pm 0.87^{* *}$ \\
$\mathrm{AUC}_{\text {total }}(\mu \mathrm{g}$. hour$/ \mathrm{ml})$ & $41.3 \pm 0.53$ & $36.3 \pm 1.03^{*}$ & $33.2 \pm 0.93^{* *}$ \\
$t_{1 / 2}($ hour$)$ & $2.62 \pm 1.12$ & $2.53 \pm 0.96^{*}$ & $2.42 \pm 1.23^{*}$ \\
$\mathrm{MRT}($ hour$)$ & $5.43 \pm 0.89$ & $4.91 \pm 1.08^{*}$ & $4.74 \pm 0.74^{*}$ \\
$V_{\mathrm{d}}(\mathrm{ml})$ & $91.4 \pm 0.52$ & $92.3 \pm 1.45$ & $93.2 \pm 0.83$ \\
$C_{\mathrm{T}}(\mathrm{ml} / \mathrm{minute})$ & $2.56 \pm 0.58$ & $2.63 \pm 2.01$ & $2.43 \pm 0.77$ \\
\hline
\end{tabular}

All values are expressed as Mean $\pm \mathrm{SD}$.

SXG = saxagliptin; SDI = single-dose interaction; $\mathrm{MDI}=$ multiple-dose interaction

*Significant with $p<0.05 ; * *$ Significant with $p<0.01$.

\section{Statistical analysis}

All the obtained results were expressed as mean $\pm \mathrm{SD}$. One-way analysis of variance was used to statistically evaluate the obtained data using the Graphpad Prism 7.01 software. Values with $p<0.05$ were considered as statistically significant.

\section{RESULTS}

\section{HPLC analysis}

The limit of detection (LOD) and limit of quantification (LOQ) of the analysis were found to be $0.27455 \mu \mathrm{g}$ and 0.87422 $\mu \mathrm{g}$, respectively.

\section{Pharmacokinetic interaction study in normal rats}

The calculated pharmacokinetic parameters are represented in Table 1. $C_{\max }$ was significantly decreased by $9.16 \%$ (SDI group) and $11.72 \%$ (MDI group) in rutin pretreated group compared to the control (Saxagliptin alone) group. $\mathrm{AUC}_{0-\mathrm{n}}$ was decreased by $19.9 \%$ (SDI group) and $27.04 \%$ (MDI group) and $\mathrm{AUC}_{\text {total }}$ was also decreased by $12.1 \%$ (SDI group) and $19.61 \%$ (MDI group) in rutin pretreated groups compared to the control group. $T_{\max }$ was observed after 2.0 hours of drug administration in to body. MRT and $t_{1 / 2}$ were also decreased significantly in rutin pretreated groups. Parameters like $V_{\mathrm{d}}$ and $C l_{\mathrm{T}}$ were slightly modified in pretreated groups.

The serum drug concentration profiles of the three treated groups of normal rats are shown in Figure 1.

\section{Pharmacokinetic interaction study in diabetic rats}

The calculated pharmacokinetic parameters are represented in Table 2. $C_{\max }$ was significantly decreased by $10.97 \%$ (SDI group) and $23.4 \%$ (MDI group) in rutin pretreated groups compared to the control group. $\mathrm{AUC}_{0-\mathrm{n}}$ was decreased by

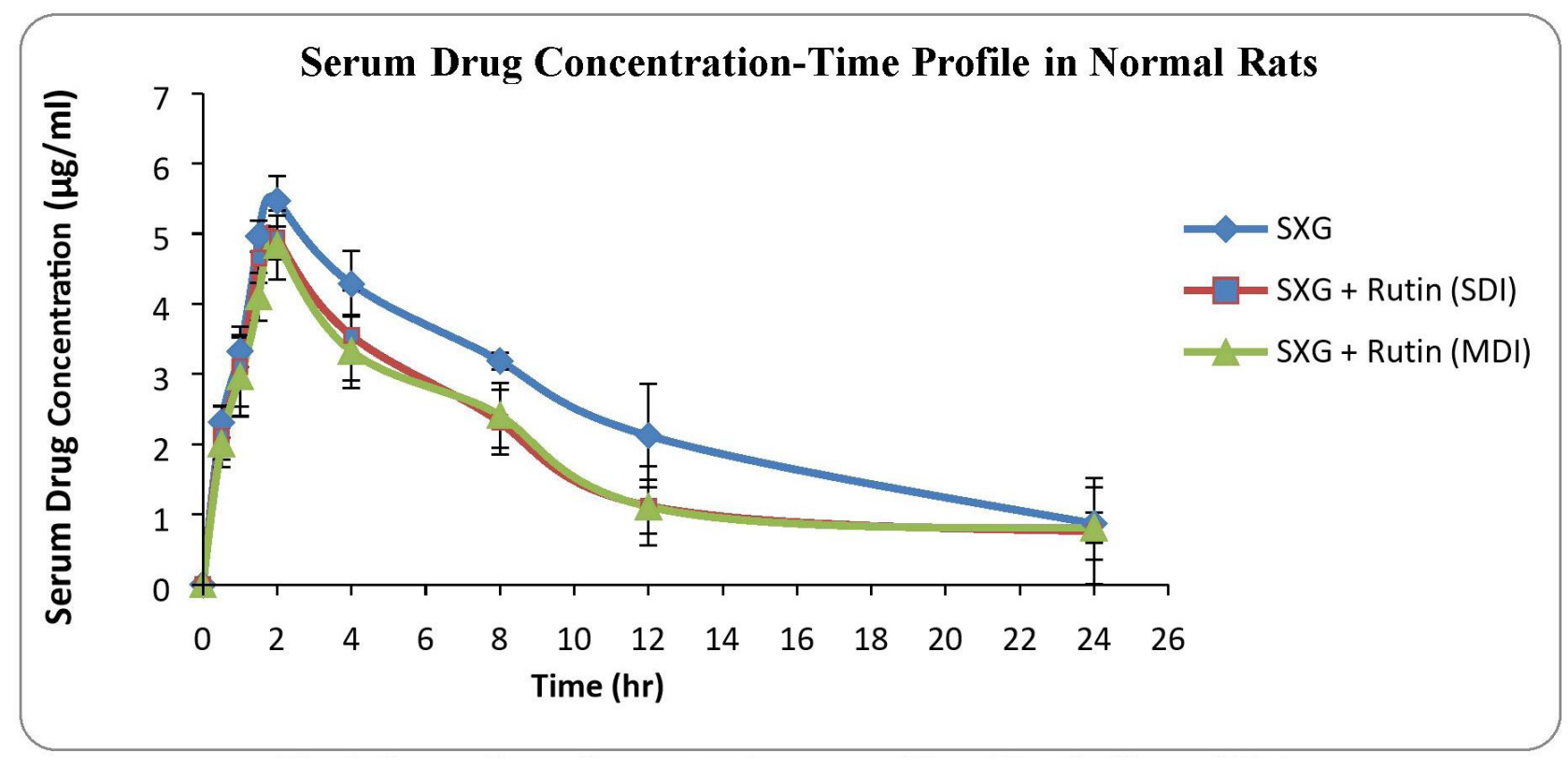

Figure 1. Serum drug concentration versus time plots in normal rats. 
$11.1 \%$ (SDI group) and 24.5\% (MDI group) and $\mathrm{AUC}_{\text {total }}$ was also decreased by $10.81 \%$ (SDI group) and $26.2 \%$ (MDI group) in rutin pretreated groups compared to the control group. $T_{\max }$ was observed after 2.0 hours of drug administration in to body. MRT and $t_{1 / 2}$ were also decreased significantly in rutin pretreated groups. Parameters like $V_{\mathrm{d}}$ and $\mathrm{Cl}_{\mathrm{T}}$ were slightly modified in pretreated groups.

The serum drug concentration profiles of the three treated groups of diabetic rats are shown in Figure 2.

\section{Pharmacodynamic interaction study in diabetic rats}

The percentage of glucose reduction at each time point was calculated in comparison with mean glucose level at 0 time point (Table 3). The maximum glucose reduction was observed in the group treated with Saxagliptin alone compared to rutin pretreated groups. The maximum glucose reduction $(34.43 \% \pm$ $1.54 \%$ ) was observed after 8 hours of drug administration in group 1 , whereas in group 2 and 3 it was $25.56 \% \pm 2.14 \%$ and $23.90 \%$

Table 2. Pharmacokinetic parameters of Saxagliptin in diabetic rats.

\begin{tabular}{lccc}
\hline PK parameter & SXG & SXG + Rutin (SDI) & SXG + Rutin (MDI) \\
\hline$C_{\text {max }}(\mu \mathrm{g} / \mathrm{ml})$ & $6.56 \pm 0.31$ & $5.84 \pm 0.25^{*}$ & $5.02 \pm 0.27^{* *}$ \\
$T_{\max }($ hour $)$ & $2.0 \pm 0$ & $2.0 \pm 0$ & $2.0 \pm 0$ \\
$\mathrm{AUC}_{0-\mathrm{n}}(\mu \mathrm{g}$. hour$/ \mathrm{ml})$ & $43.2 \pm 1.62$ & $38.4 \pm 0.92^{*}$ & $32.6 \pm 1.17^{* *}$ \\
$\mathrm{AUC}_{\text {total }}(\mu \mathrm{g}$. hour$/ \mathrm{ml})$ & $51.8 \pm 0.54$ & $46.2 \pm 3.03^{*}$ & $38.2 \pm 1.93^{* *}$ \\
$t_{1 / 2}($ hour$)$ & $2.82 \pm 0.24$ & $2.73 \pm 0.72^{*}$ & $2.64 \pm 0.73^{*}$ \\
$\mathrm{MRT}($ hour$)$ & $6.36 \pm 0.79$ & $5.93 \pm 1.28^{*}$ & $4.94 \pm 0.64^{*}$ \\
$V_{\mathrm{d}}(\mathrm{ml})$ & $88.4 \pm 0.72$ & $87.3 \pm 2.45$ & $88.2 \pm 0.63$ \\
$C_{\mathrm{T}}(\mathrm{ml} / \mathrm{minute})$ & $2.16 \pm 0.48$ & $2.33 \pm 1.51$ & $2.31 \pm 0.77$ \\
\hline
\end{tabular}

All values are expressed as Mean $\pm \mathrm{SD}$;

$\mathrm{SXG}=$ saxagliptin; $\mathrm{SDI}=$ single-dose interaction; $\mathrm{MDI}=$ multiple-dose interaction

*Significant with $p<0.05$; **Significant with $p<0.01$. $\pm 1.84 \%$, respectively. The maximum glucose reduction was observed after 12 hours of drug administration in rutin pretreated groups (Fig. 3).

\section{DISCUSSION}

The developed HPLC method was found to have accuracy and precision. The estimated LOD and LOQ were found to be within the range of the analyzed levels in serum samples (Jyothi et al., 2017). So, the developed chromatographic method for drug estimation has been proved to give the best results with good sensitivity.

The decrease in $C_{\text {max }}$ and AUC values in rutin pretreated rats in both normal and diabetic groups clearly indicated that the bioavailability of Saxagliptin was decreased. It may be because of the induction of CYP 3A4 activity by rutin, which resulted in an

Table 3. Pharmacodynamic parameters in diabetic rats.

\begin{tabular}{cccc}
\hline \multirow{2}{*}{ Time (hour) } & \multicolumn{3}{c}{ Mean glucose level (mg/dl) } \\
\cline { 2 - 4 } & SXG & SXG + Rutin (SDI) & SXG + Rutin (MDI) \\
\hline 0 & $284.23 \pm 2.12$ & $279.24 \pm 2.03$ & $275.41 \pm 0.83$ \\
0.5 & $271.32 \pm 1.12$ & $268.21 \pm 1.46$ & $270.34 \pm 1.65$ \\
1 & $251.14 \pm 0.96$ & $261.16 \pm 1.75$ & $263.85 \pm 2.74^{* *}$ \\
1.5 & $236.13 \pm 0.78$ & $248.32 \pm 2.37^{*}$ & $254.65 \pm 2.74^{* *}$ \\
2 & $224.65 \pm 1.89$ & $239.19 \pm 2.89^{*}$ & $243.55 \pm 1.35^{* *}$ \\
4 & $198.54 \pm 1.54$ & $231.56 \pm 3.45^{*}$ & $231.45 \pm 1.78^{*}$ \\
8 & $186.37 \pm 1.45$ & $219.25 \pm 1.17^{*}$ & $214.12 \pm 0.93^{*}$ \\
12 & $188.79 \pm 0.95$ & $207.86 \pm 0.86^{*}$ & $209.58 \pm 2.45^{*}$ \\
24 & $187.24 \pm 2.34$ & $208.12 \pm 1.87$ & $211.35 \pm 1.67$ \\
\hline
\end{tabular}

All values are expressed as Mean $\pm \mathrm{SD}$.

SXG = saxagliptin; SDI = single-dose interaction; $\mathrm{MDI}=$ multiple-dose interaction *Significant with $p<0.05$; * Significant with $p<0.01$.

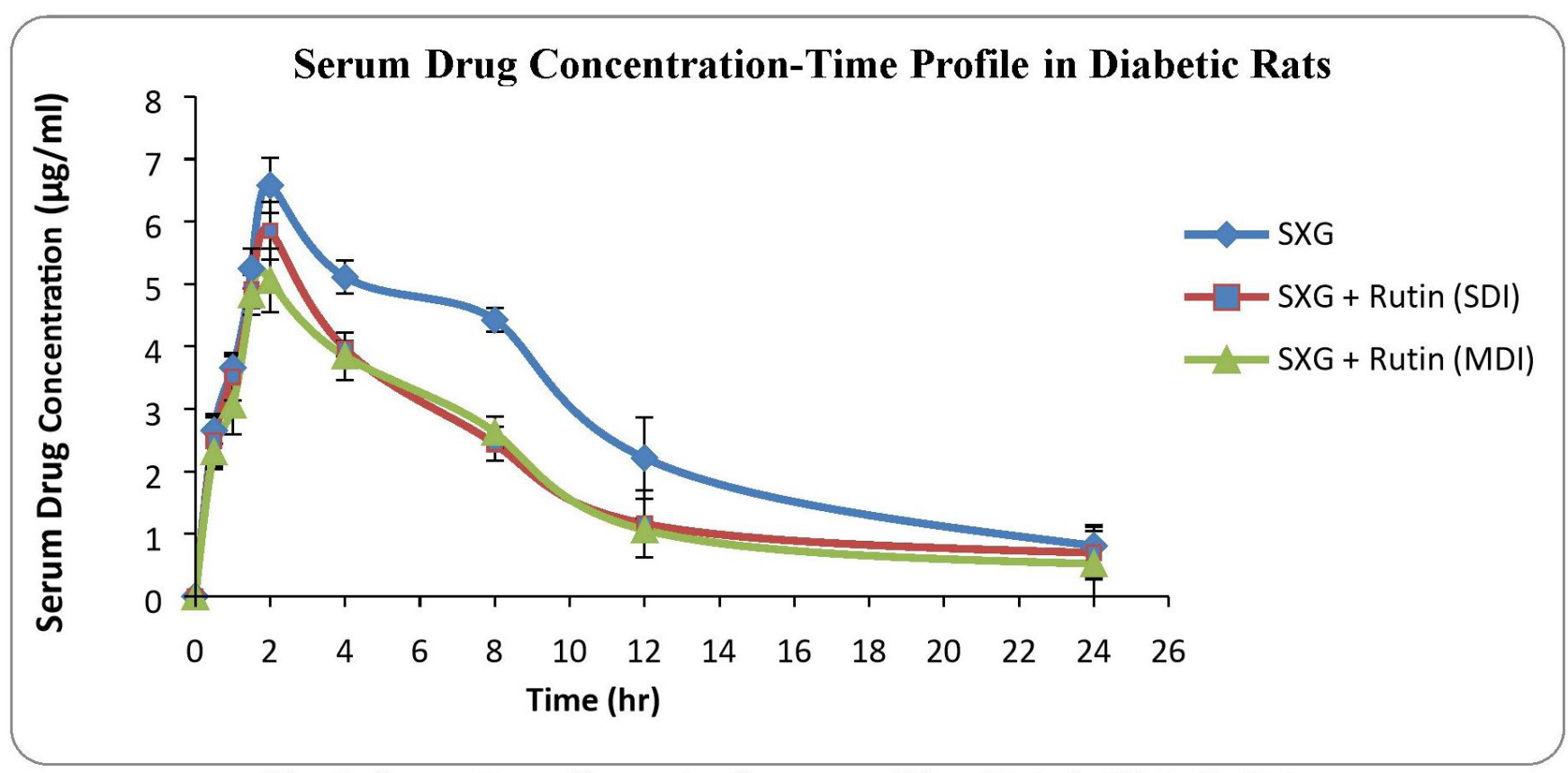

Figure 2. Serum drug concentration versus time plots in diabetic rats. 


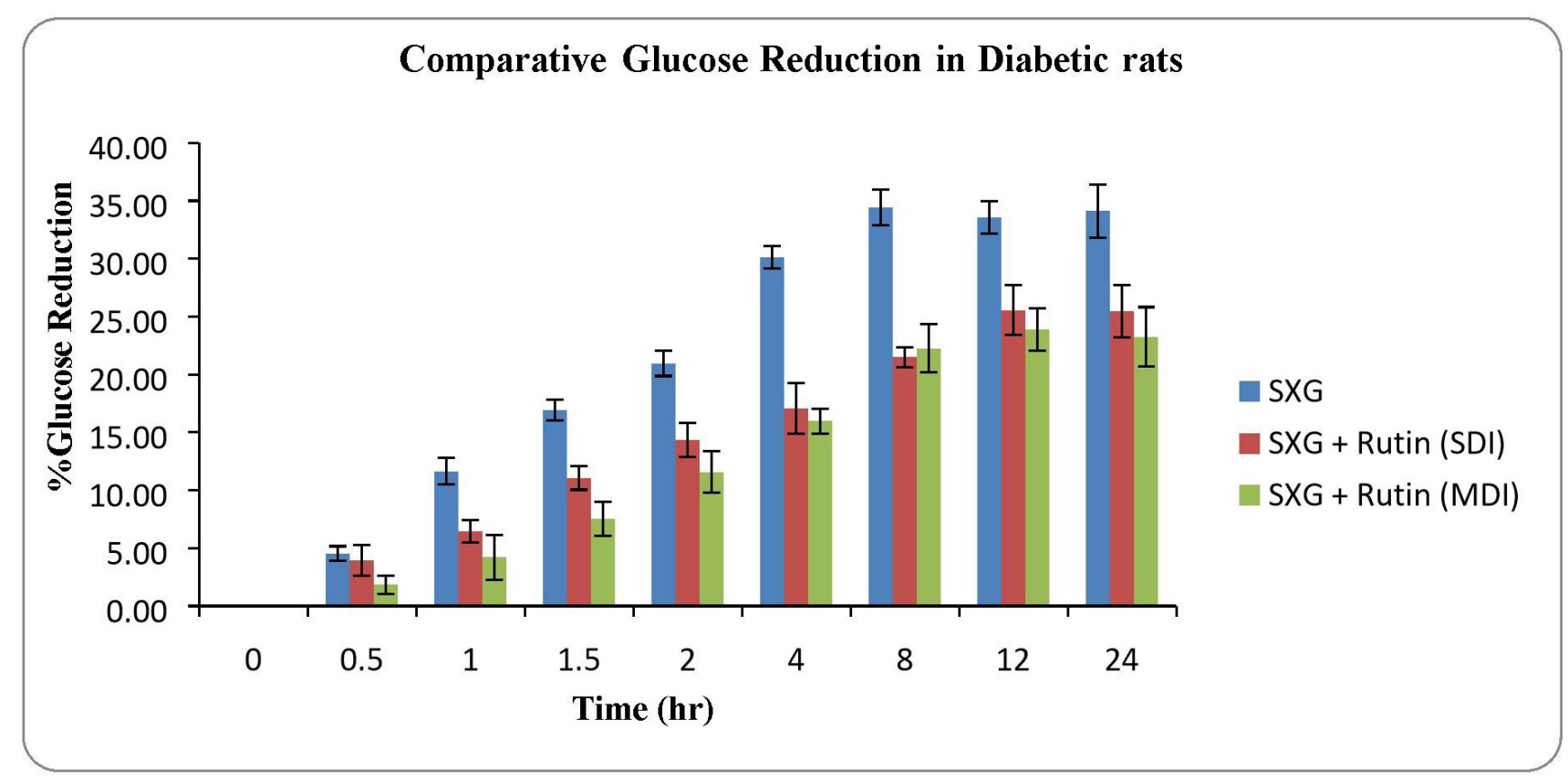

Figure 3. Glucose reduction profile in diabetic rats.

increased metabolism of Saxagliptin (Ganeshpurkar and Saluja, 2017). It is clearly understood that the bioavailable drug fraction was decreased due to the rise in drug metabolism. $T_{\max }$ was not altered in rutin pretreated groups. This indicated that the rate of drug absorption was not affected in the presence of rutin. $t_{1 / 2}$ and MRT were slightly decreased in rutin pretreated groups compared to the control group. It may be due to increased drug metabolism which reduced the drug amount in body fluids, thereby reducing drug residence period in the body (Devi et al., 2015). There was a negligible alteration in volume of distribution and total body clearance in rutin pretreated groups, which indicated that rutin could not affect the protein binding nature of the drug with body fluid proteins (Vatsavai and Kilari, 2016; Chaudhari et al., 2019). The alterations in pharmacokinetic parameters were more significant in MDI rutin pretreated groups in both normal and diabetic rats.

The decreased glucose reductions were observed in rutin pretreated rats. This may be because of decreased pharmacokinetic parameters of Saxagliptin (Jyothi et al., 2017; Neearti, 2012). It is clearly understood that the increased activity of CYP $3 A 4$ due to presence of rutin had greatly improved the drug metabolism which resulted in a decreased active drug fraction in body fluids. This resulted in the reduction of antihyperglycemic activity of Saxagliptin. The effect of rutin on antidiabetic property of Saxagliptin was more significant in the MDI group compared to the SDI group.

\section{CONCLUSION}

The phytochemical rutin had greatly interfered with the extent of pharmacokinetic and pharmacodynamic parameters. Rutin, as an inducer of CYP 3A4, was responsible for the increased metabolism of Saxagliptin, thereby reducing the drug's bioavailable fraction. Due to the decrease of $C_{\max }$ and AUC of drug in the presence of rutin, the antidiabetic activity of Saxagliptin has been reduced. From the results of this research study, it can be concluded that the rutin has shown significant interactions with Saxagliptin and has altered its therapeutic activity. So, it is advised to be away from taking vegetables, fruits, and ayurvedic products containing rutin while the patient is on antidiabetic therapy with Saxagliptin. This research study reveals the importance of dose adjustment of Saxagliptin, if it is to be administered along with rutin.

\section{ACKNOWLEDGMENTS}

The authors are thankful to the Glenmark Pharmaceuticals Limited, Mumbai, for supplying the required drug samples for research work.

\section{CONFLICT OF INTEREST}

All the authors involved in this research work declare no conflict of interest for the results of study and publication of the manuscript.

\section{FUNDING}

None.

\section{REFERENCES}

Aba PE, Asuzu1 IU. Mechanisms of actions of some bioactive anti-diabetic principles from phytochemicals of medicinal plants: a review. Indian J Nat Prod Resour, 2018; 9(2):85-96.

Chaudhari S, Zambad S, Ali M. Effect of aqueous extract of azadirachta indica leaves on pharmacokinetics and pharmacodynamics of glipizide. Drug Metab Lett, 2019; 13(1): 19-24.

Devi PRS, Reddy AG, Rao GS, Kumar CSVS, Boobalan G Pharmacokinetic interaction of curcumin and glibenclamide in diabetic rats. Vet World, 2015; 8:508-11. 
Gaikwad SB, Mohan GK, Rani MS. Phytochemicals for diabetes management. Pharm Crops, 2014; 5(Suppl 1: M2):11-28.

Ganeshpurkar A, Saluja AK. The pharmacological potential of rutin. Saudi Pharm J, 2017; 25:149-64.

Gao J, Shi Z, Zhu S, Li GQ, Yan R, Yao M. Influences of processed rhubarbs on the activities of four CYP isozymes and the metabolism of Saxagliptin in rats based on probe cocktail and pharmacokinetics approach. J Ethanopharmacology, 2013; 145(2):566-72.

Jyothi P, Thirupathi G, Narsimha Reddy Y. Influence of diosgenin on pharmacokinetics and pharmacodynamics of repaglinide in rats. Int J Pharm Biol Sci, 2017; 7(1):101-8.

Neearti P. Influence of curcumin on pioglitazone metabolism and Pk/Pd: diabetes mellitus. J Diabetes Metab, 2012; S6-003:1-6.

Sowjanya CH, Rama RA, Veeresham C. Pharmacokinetic and pharmacodynamic interaction of Quercetin with Saxagliptin in normal and diabetic rats. Pharmacologia, 2017; 8(3):90-4.

Vatsavai LK, Kilari EK. Influence of curcumin on the pharmacodynamics and pharmacokinetics of gliclazide in animal models. J Exp Pharmacol, 2016; 8:69-76.
Wanwimolruk S, Prachayasittikul V. Cytochrome P450 enzyme mediated herbal drug interactions (Part 1). EXCLI J, 2014; 13:347-91.

$\mathrm{Yu} \mathrm{CP}, \mathrm{Wu} \mathrm{PP}, \mathrm{LC} \mathrm{PD}$. Quercetin and rutin reduced the bioavailability of cyclosporine from neural, an immunosuppressant, through activating P-glycoprotein and CYP 3A4. J Agric Food Chem, 2011; 59:4644-8.

\section{How to cite this article:}

Kandukoori NR, Uppu P, Yellu NR. Study of alterations in pharmacokinetics and pharmacodynamics of Saxagliptin in the presence of Rutin: An interaction study in rats. J Appl Pharm Sci, 2020; 10(11):081-086. 